\title{
Vibrational Levels Associated with Hydrogen Bonds and Semiclassical Hamiltonian Normal Forms
}

\author{
George A. Hagedorn and Alain Joye
}

\author{
Dedicated to Jean-Michel Combes, in celebration of his $65^{\text {th }}$ birthday
}

\begin{abstract}
We describe and extend our recent proposal to model mathematically the vibrational levels associated with hydrogen bonds in symmetric triatomic molecules. Our approach is based on modification of the usual BornOppenheimer approximation to take into account the lighter mass of the hydrogen nucleus and the weakness of the hydrogen bond, using special features of the electron energy level surface associated with the hydrogen bond. Neglecting bending of the molecule for simplicity, we achieve this by scaling the mass of the hydrogen atoms differently from the heavier atoms, and by using a modified form for the electronic energy surface.

As a result, anharmonic effects play a role at leading order in the limit where the nuclear masses go to infinity. Our analysis is based on close examination of the numerical data available for the ground state energy surface of the $F H F^{-}$ion, and we make a comparison with experimental data for the vibrational levels of that ion.

The theory we propose is, however, quite general and can accomodate asymmetric tri-atomic molecules. Moreover, we provide an extension of our results to molecules with nuclei of several different species, where we assume that each of the masses scales differently. Considering an adapted ground state energy surface, we compute the leading term of the corresponding vibrational levels in the limit of large nuclear masses by means of a normal form Hamiltonian.
\end{abstract}

\section{Introduction}

The standard time-independent Born-Oppenheimer approximation [1] takes advantage of the large masses of the nuclei relative to the mass of an electron. It allows one to compute the low-lying vibrational states of the nuclear motion from knowledge of the ground state electron energy level surface near its minimum under the assumption that the minimum is non-degenerate. To leading order, the vibrational energy levels are those of a harmonic oscillator associated with the non-degenerate minimum.

1991 Mathematics Subject Classification. 81V55.

Key words and phrases. Hydrogen bonds, vibrational levels, Born-Oppenheimer approximation, semiclassical normal forms.

Partially Supported by National Science Foundation Grants DMS-0303586 and DMS0600944. 
More precisely, consider a molecular system whose nuclear configuration space modulo rotations is $\mathbb{R}^{d}$, and denote the ratio of mean of the nuclear masses to the electron mass by $\epsilon^{-4}$. For small $\epsilon$, there exists a molecular energy level $\mathcal{E}(\epsilon)$ that satisfies,

$$
\mathcal{E}(\epsilon)=E_{0}+\epsilon^{2}\left(\left(n_{1}+\frac{1}{2}\right) \omega_{1}+\cdots\left(n_{d}+\frac{1}{2}\right) \omega_{d}\right)+O\left(\epsilon^{4}\right),
$$

where $E_{0}$ is the minimum of the ground state electronic energy level. The numbers $n_{j} \in \mathbb{N}$, and $\omega_{j}>0$, for $j=1, \cdots, d$, characterize the $d$-dimensional harmonic oscillator. Under quite general hypotheses, (1.1) represents the first few terms in a complete asymptotic expansion of $\mathcal{E}(\epsilon)$ in powers of $\epsilon$. See the recent review [4] for more details and results.

For many molecules, this approximation is very successful, but it may fail to give accurate results when applied to some molecules that contain hydrogen bonds. The binding energy of hydrogen bonds is typically very small, and the mass of the hydrogen nucleus is an order of magnitude smaller than that of other nuclei such as carbon. Moreover, the experimental vibrational spectra of symmetric tri-atomic molecules with hydrogen bonds display significant deviations from the approximate harmonic spectrum (1.1). See [2] for an account of these properties.

In $[\mathbf{3}]$ we propose an alternative to the Born-Oppenheimer approximation that is specifically designed to describe molecules with symmetrical hydrogen bonds. There are two main differences between our approach and the standard BornOppenheimer analysis. First, we scale the masses of the hydrogen nuclei as $\epsilon^{-3}$ while keeping the heavier nuclei scale as $\epsilon^{-4}$. Although our analysis applies only for small values of $\epsilon$, the mass of a hydrogen nucleus is $1.015 \epsilon^{-3}$ if $\epsilon$ is defined by taking the mass of a carbon atom to be $\epsilon^{-4}$. This value of $\epsilon$ is roughly $\epsilon \simeq 0.082$. Second, we model the electron energy level surface in a special way that depends on $\epsilon$. This takes into account the smallness of a coefficient in the harmonic potential associated with the hydrogen bond. That coefficient is weaker than the others, roughly by a factor $\epsilon$. Consequently, in our model, anharmonic effects contribute to the leading order calculations of vibrational levels, as $\epsilon \rightarrow 0$.

Our approach gives rise to an $\epsilon$-independent normal form Hamiltonian $H_{N F}$ (described below) which is not a harmonic oscillator. This Hamiltonian describes the vibrational levels: For small $\epsilon$, there exists a molecular level $\mathcal{E}(\epsilon)$ that satisfies

$$
\mathcal{E}(\epsilon)=E_{0}+\epsilon^{2} \mathcal{E}_{2}+O\left(\epsilon^{2+\xi}\right),
$$

for some $\xi>0$, where $\mathcal{E}_{2}$ is an element of the spectrum of $H_{N F}$. Note that the index 2 refers here to the power of $\epsilon$ appearing as the leading term. Again, under appropriate hypotheses, our analysis yields a complete expansion of $\mathcal{E}(\epsilon)$ in powers of $\epsilon^{1 / 2}$.

Our model leads to a different expansion from the usual Born-Oppenheimer approximation. For hydrogen nuclei not involved in hydrogen bonding, the vibrational energies are of order $\epsilon^{3 / 2}$, while the vibrational energies for the other nuclei and the hydrogen nuclei involved in the symmetric hydrogen bonding are of order $\epsilon^{2}$. Furthermore, anharmonic effects must be taken into account for a hydrogen nucleus involved in hydrogen bonding at the leading order, $\epsilon^{2}$. In the standard Born-Oppenheimer model, all vibrational energies appear at order $\epsilon^{2}$ and are harmonic at that order. Anharmonic corrections first enter at order $\epsilon^{4}$. 
Our analysis is motivated by an examination of symmetric bihalide ions, such as $\mathrm{FHF}^{-}$or $\mathrm{ClHCl}^{-}$, and we work under the simplifying hypothesis that bending of the molecule is absent. Although symmetric bihalide ions are quite special, our approach is flexible enough to describe more general phenomena. For example, the lowest electron energy surface for $F H F^{-}$has a single minimum with the hydrogen nucleus mid-way between the two fluorines. Our general theory can handle situations with single or closely spaced double wells in the coordinates for a hydrogen nucleus that participates in hydrogen bonding.

In this note, we further generalize our approach to molecules with nuclei of several different species, where we assume that each of the masses scales with a different inverse power of $\epsilon$. We allow the ground state energy surface to depend on $\epsilon$ in a similar fashion as in the tri-atomic case, and we determine a corresponding normal form $H_{N F}$. Then, we prove that the leading term of the vibrational levels $\mathcal{E}(\epsilon)$ is described by $H_{N F}$ :

$$
\mathcal{E}(\epsilon)=\epsilon^{\sigma} \mathcal{E}_{\sigma}+O\left(\epsilon^{\sigma+\xi}\right)
$$

for small $\epsilon$, where

$$
\mathcal{E}_{\sigma} \in \sigma\left(H_{N F}\right), \quad \sigma>0, \quad \text { and } \quad \xi>0 .
$$

Here, the index $\sigma$ refers to the power of $\epsilon$ in the leading term.

From a mathematical point view, our analysis consists of constructing and studying quasimodes for the full molecular Hamiltonian in the singular limit $\epsilon \rightarrow 0$. To do this, we use a multiscale analysis technique. We identify different relevant length scales in the system and treat them independently in the first stage of the analysis. This leads to a formal algorithm to construct an Ansatz for the quasimode in more variables. We then prove that this yields a bona fide quasimode for the original problem by re-introducing the coupling between the length scales. This is a traditional, successful route for getting information on the vibrational spectrum of the molecule. See e.g., [4]. In the present circumstances, when using the same strategy, we face a new problem: The spectrum of the normal form Hamiltonian $H_{N F}$ is not known explicitly, and we have to show that the eigenfunctions of $H_{N F}$ and their derivatives have sufficient decay properties. For the harmonic oscillator of the standard Born-Oppenheimer theory, everything required is well-known.

\section{The Tri-Atomic Model}

We consider a molecule that consists of two types of atoms. Two identical nuclei are heavier and have masses $\epsilon^{-4}$, where the mass of an electron is 1 . The third nucleus is lighter and has mass $\epsilon^{-3}$. We consider a simplified model in which bending and rotational motions are absent, i.e., all three nuclei lie on a fixed line. The two heavier nuclei have coordinates $q_{1}$ and $q_{2}$ whereas the coordinate of the lighter nucleus is $q_{3}$. Then we pass to Jacobi coordinates and discard the kinetic energy of the center of mass. For convenience, we also rescale coordinates by constant numerical factors and neglect some harmless $\epsilon$ dependent mass correction, (See $[\mathbf{3}]$ ), to get a molecular Hamiltonian of the form,

$$
H_{\mathrm{mol}}(\epsilon)=-\frac{\epsilon^{4}}{2} \partial_{x_{1}}^{2}-\frac{\epsilon^{3}}{2} \partial_{x_{2}}^{2}+h\left(x_{1}, x_{2}\right),
$$

where $x_{1}=\left(q_{2}-q_{1}\right) / \sqrt{2}$ denotes (essentially) the distance between the two identical nuclei, and $x_{2}=q_{3}-\left(q_{1}+q_{2}\right) / 2$ is the dispacement of the lighter nucleus from the 
center of mass of the identical nuclei. Further technical hypotheses are provided below in Assumption 3.1.

The electronic Hamiltonian $h\left(x_{1}, x_{2}\right)$ depends parametrically on the positions of the nuclei. It is a self-adjoint operator on the electronic Hilbert space $\mathcal{H}_{e l}$. We assume $h\left(x_{1}, x_{2}\right)$ has a non-degenerate ground state electronic surface $E\left(x_{1}, x_{2}\right)$ with corresponding normalized eigenvector $\Phi\left(x_{1}, x_{2}\right)$. We further assume that the molecule actually binds, in the sense that that there exists a nuclear configuration $\left(x_{1}^{e q}, x_{2}^{e q}\right)$ that is an isolated minimum of $E\left(x_{1}, x_{2}\right)$. We assume $x_{1}^{e q} \neq 0$. (The heavy nuclei are not at the same point!) In the symmetrical case which we study here, the equilibrium position of the lighter nucleus lies at the center of mass of the heavy nuclei $\left(x_{2}^{e q}=0\right)$. The non-symmetrical case would have $x_{2}^{e q} \neq 0$.

Thus far, we have only altered the standard Born-Oppenheimer approximation by introducing two nuclear mass scales, instead of one. We now make a second modification to reflect the structure of the electron energy level surface associated with hydrogen bonding. (See [2].) We introduce an artificial dependence in the ground state electronic surface on $\epsilon$. After a shift of the variable $x_{1}$ by $x_{1}^{e q}$, we assume that the ground state has an expansion of the form

$$
E\left(\epsilon, x_{1}, x_{2}\right)=E_{0}+a_{1} x_{1}^{2}+a_{2} \epsilon x_{2}^{2}-a_{3} x_{1} x_{2}^{2}+a_{4} x_{2}^{4}+O\left(x_{1}^{\nu_{1}} x_{2}^{2 \nu_{2}}\right),
$$

where $\nu_{1}, \nu_{2} \in \mathbb{N}$ and $\nu_{1}+\nu_{2} \geq 3$. The Taylor series only contains even powers of $x_{2}$, due to the symmetry of the molecule. The factor $\epsilon$ in the harmonic term $a_{2} \epsilon x_{2}^{2}$ expresses the weaker binding of the hydrogen atom. The smallness of this coefficient makes it necessary to consider terms of order higher than quadratic in the expansion. We assume that $a_{1}, a_{3}$ and $a_{4}$ are positive, but $a_{2}$ can take any value. When $a_{2}$ is negative, $E\left(\epsilon, x_{1}, x_{2}\right)$ has a closely spaced double well near $\left(x_{1}^{e q}, 0\right)$, instead of a single local minimum. Note that $a_{3}=0$ leads to two decoupled onedimensional systems, to leading order. We define then a scalar normal form by keeping the leading order terms only in (2.1),

$$
-\frac{\epsilon^{4}}{2} \partial_{x_{1}}^{2}-\frac{\epsilon^{3}}{2} \partial_{x_{1}}^{2}+E_{0}+a_{1} x_{1}^{2}+a_{2} \epsilon x_{2}^{2}-a_{3} x_{1} x_{2}^{2}+a_{4} x_{2}^{4}
$$

The Schrödinger operator (2.2) plays the role the Harmonic oscillator plays in the standard Born-Oppenheimer approximation. By rescaling with $\left(y_{1}, y_{2}\right)=$ $\left(x_{1} / \epsilon, x_{2} / \epsilon^{1 / 2}\right)$, we see that (2.2) minus the constant term $E_{0}$ is unitarily equivalent to $\epsilon^{2}$ times the normal form Hamiltonian $H_{N F}$,

$$
H_{N F}=-\frac{1}{2} \partial_{y_{1}}^{2}-\frac{1}{2} \partial_{y_{2}}^{2}+E_{N F}\left(y_{1}, y_{2}\right)
$$

with

$$
E_{N F}\left(y_{1}, y_{2}\right)=a_{1} y_{1}^{2}+a_{2} y_{2}^{2}-a_{3} y_{1} y_{2}^{2}+a_{4} y_{2}^{4}
$$

We further assume that the coefficients $\left\{a_{j}\right\}$ are such that the normal form potential $E_{N F}$ is bounded below. More precisely, we require

Assumption 2.1.

and either

$$
a_{1}, a_{3}, a_{4}>0
$$

$$
a_{3}^{2}<4 a_{1} a_{4},
$$

or

$$
a_{3}^{2}=4 a_{1} a_{4} \text { and } a_{2} \geq 0 \text {. }
$$


Anticipating the result of the analysis below, the normal form $H_{N F}$ (2.3) yields the leading term asymptotics (1.2) for the vibrational levels.

The special form (2.1) is motivated by numerical data for the molecule $F H F^{-}$. By fitting numerical data for $F H F^{-}$with the value $\epsilon=0.0821$, we get the following quantities, where distances are expressed in Angströms and energies in Hartrees.

$$
\begin{aligned}
& x_{1}^{e q}=1.617, \quad E_{0}=-200.215, \\
& a_{1}=0.52, \quad a_{2}=1.22, \quad a_{3}=1.82, \quad a_{4}=1.62 .
\end{aligned}
$$

The experimentally observed values [2] for the lowest symmetric stretch, asymmetric stretch, and combined vibrational modes are, respectively,

$$
583.05 \mathrm{~cm}^{-1}, \quad 1331.15 \mathrm{~cm}^{-1} \text {, and } 1849 \mathrm{~cm}^{-1} \text {. }
$$

A mode is defined as the difference between an excited state eigenvalue and the ground state eigenvalue. The first mode corresponds to the situation where the heavy fluorine nuclei oscillate symmetrically whereas the hydrogen nuclei remains fixed. The second mode corresponds to the situation where the lighter hydrogen nucleus and the fluorine nuclei oscillate while the distance between the fluorine nuclei remains fixed. The third mode corresponds to a mixture of these two types of motion.

Note that the sum of the first two modes is significantly larger than the third one. This is an expression of anharmonicity, since in the harmonic approximation, these three modes would be given by $\epsilon^{2} \omega_{1}, \epsilon^{2} \omega_{2}$ and $\epsilon^{2}\left(\omega_{1}+\omega_{2}\right)$, respectively, see (1.1).

By comparison, if we use the data (2.4) in $H_{N F}$, and numerically determine the vibrational levels using the first two terms of (1.2) with $\epsilon=0.0821$, i.e.

$$
\mathcal{E}(\epsilon) \simeq E_{0}+\epsilon^{2} \mathcal{E}_{2}, \quad \text { with } \quad \mathcal{E}_{2} \in \sigma\left(H_{N F}\right),
$$

our model predicts the following values for these vibrational modes:

$$
600 \mathrm{~cm}^{-1}, \quad 1399 \mathrm{~cm}^{-1} \text {, and } 1942 \mathrm{~cm}^{-1} \text {. }
$$

To get these three modes, it is necessary to compute numerically the first six energy levels of $H_{N F}$ as well as the corresponding eigenvectors in order to attribute the levels to the relevant motions of the nuclei. More precisely, the modes correspond successively to $\epsilon^{2}\left(E_{1}-E_{0}\right), \epsilon^{2}\left(E_{3}-E_{0}\right)$ and $\epsilon^{2}\left(E_{5}-E_{0}\right)$, where $E_{j}$ is the $j$ th level of $H_{N F}$.

Besides the relatively good agreement with the the experimental data, we also point out that the anharmonic effect mentioned above is present in our leading term computation.

Standard Born-Oppenheimer based software yields corresponding harmonic modes

$$
609 \mathrm{~cm}^{-1}, \quad 1118 \mathrm{~cm}^{-1} \text {, and } 1727 \mathrm{~cm}^{-1} \text {. }
$$

Encouraged by this positive numerical test, we turn to the mathematical proof of assertion (1.2). It is a special case of the generalization (1.3), which we describe in the next section. 


\section{More mass scales}

The tri-atomic model presented in [3] and its mathematical analysis generalize naturally. Roughly speaking, we can consider molecules with nuclei of several different species, where we assume that each of the masses scales with a distinct inverse power of $\epsilon$. The corresponding ground state energy surface depends on $\epsilon$ in a similar way to the tri-atomic case.

We adopt this more general point of view here and prove the leading order result (1.3) which generalizes (1.2). More precisely, we work under the following general assumption.

Assumption 3.1. Let $H_{\text {mol }}(\epsilon)$ be a self-adjoint molecular Hamiltonian defined on a domain of $L^{2}\left(\mathbb{R}^{n}, \mathcal{H}_{e l}\right)$ by

$$
H_{\mathrm{mol}}(\epsilon)=\sum_{j=1}^{n}-\frac{\epsilon^{\mu_{j}}}{2} \partial_{x_{j}}^{2}+h(\epsilon, x), \quad \mu_{1} \geq \mu_{2} \geq \cdots \geq \mu_{n}>0 .
$$

Here, $x \in \mathbb{R}^{n}$, and $\mathcal{H}_{e l}$ is the electronic Hilbert space. The electron Hamiltonian $h(\epsilon, x)$ is self-adjoint on an $x$-independent dense domain $\mathcal{D} \subset \mathcal{H}_{e l}$. We assume $h(\epsilon, x)$ is bounded below, uniformly for $x \in \mathbb{R}^{n}$. Moreover, $h(\epsilon, x)$ is $C^{\infty}$ in the strong resolvent sense and admits a non-degenerate $\epsilon$-dependent ground state eigenvalue $V(\epsilon, x)$ with corresponding normalized eigenstate $\Phi(x)$, independent of $\epsilon$. We normalize the ground state energy surface so that

$$
V(\epsilon, 0)=0 \text {. }
$$

REMARK 3.2. Our hypothesis that $\Phi(x)$ be independent of $\epsilon$ reflects the fact that the phenomenon we discuss takes place on one electronic level only. In other words, variations in the transitions between electronic levels are inessential since they contribute to higher orders only. They are ignored here for simplicity and we only put the $\epsilon$ dependence in the electron energy, not the electron state.

We denote the formal Taylor expansion of $V(\epsilon, x)$ by

$$
V(\epsilon, x) \sim \sum_{0 \neq \beta \in \mathbb{N}^{n}} v_{\beta}(\epsilon) x^{\beta}, \quad \beta=\left(\beta_{1}, \cdots, \beta_{n}\right) \in \mathbb{N}^{n}, \quad v_{\beta}(\epsilon) \in \mathbb{R},
$$

where the coefficients $v_{\beta}(\epsilon)$ are regular as $\epsilon \rightarrow 0$. We impose more restrictions on these coefficients below.

The first step in analyzing the spectrum of (3.1) consists of defining a scalar normal form Hamiltonian $H_{N F}$. Let $\alpha \in\left(\mathbb{R}^{+}\right)^{n}$ be a set of positive exponents. We consider the kinetic energy and introduce rescaled variables $\left(y_{1}, \cdots, y_{n}\right)=$ $\left(x_{1} / \epsilon^{\alpha_{1}}, \cdots, x_{n} / \epsilon^{\alpha_{n}}\right)$, which we denote by $y=x / \epsilon^{\alpha}$.

$$
\sum_{j=1}^{n}-\frac{\epsilon^{\mu_{j}}}{2} \partial_{x_{j}}^{2}=-\frac{\epsilon^{\sigma}}{2} \Delta_{y}, \quad \text { for some } \quad \sigma>0 .
$$

This enforces the relations

$$
\mu_{j}-2 \alpha_{j}=\sigma, \quad \forall j=1, \cdots, n .
$$

To determine the normal form potential $V_{N F}$, we consider the formal expression

$$
\epsilon^{-\sigma} V\left(\epsilon, \epsilon^{\alpha} y\right) \sim \sum_{0 \neq \beta \in \mathbb{N}^{n}} v_{\beta}(\epsilon)\left(\epsilon^{1 / 2}\right)^{\beta \cdot \mu-\sigma(|\beta|+2)} y^{\beta}
$$


where we use the notation $\beta \cdot \mu=\sum_{j=1}^{n} \beta_{j} \mu_{j}$ and $|\beta|=\sum_{j=1}^{n} \beta_{j}$. As in the tri-atomic case, we want $V_{N F}$ to consist of the $\epsilon^{0}$ terms on the right hand side of (3.4), and we want there to be only finitely may such terms. All other terms should contain factors of $\epsilon^{p}$ with $p$ positive. To make this more precise, we make the following assumption:

Assumption 3.3. There exist $\sigma>0$ and two non-empty finite sets of multiindices, $\beta_{0} \subset \mathbb{N}^{n} \backslash\{0\}$ and $\beta_{F} \subset \mathbb{N}^{n} \backslash \beta_{0}$, such that for small $|x|$ and $\epsilon$,

$$
\left|V(\epsilon, x)-\sum_{\beta \in \beta_{0}} v_{\beta}(\epsilon) x^{\beta}\right| \leq c \sum_{\beta \in \beta_{F}}\left|x^{\beta}\right|
$$

for some $c>0$. Furthermore,

$$
\left\{\begin{array}{c}
v_{\beta}(\epsilon)\left(\epsilon^{1 / 2}\right)^{\beta \cdot \mu-\sigma(|\beta|+2)}=c_{\beta}, \quad \forall \beta \in \beta_{0} \\
\beta \cdot \mu-\sigma(|\beta|+2) \quad>0, \quad \forall \beta \in \beta_{F} .
\end{array}\right.
$$

REMARK 3.4. We address the question of existence of a suitable $\sigma$ and the corresponding sets $\beta_{0}$ and $\beta_{F}$ for a given general potential at the end of this section.

REMARK 3.5. The case of the tri-atomic molecule in $\mathbb{R}^{2}$ is characterized by the parameters $\mu_{1}=4, \quad \mu_{2}=3, \quad \alpha_{1}=1, \quad \alpha_{2}=1 / 2, \quad \sigma=2$, and the sets

$$
\begin{aligned}
& \beta_{0}=\{(2,0),(0,2),(1,2),(0,4)\}, \\
& \beta_{F}=\{(3,0),(2,2),(1,4),(0,6)\},
\end{aligned}
$$

for which $\beta \cdot \mu-\sigma(|\beta|+2)=2$ for all $\beta \in \beta_{F}$.

REMARK 3.6. For any $0<\sigma<\mu_{n}$, the last condition in (3.6) is always satisfied for large enough $|\beta|$, since by (3.1) and (3.3),

$$
\left.\beta \cdot \mu-\sigma(|\beta|+2) \geq\left(\mu_{n}-\sigma\right)\right)|\beta|-2 \sigma=2\left(\alpha_{n}|\beta|-\sigma\right) .
$$

The normal form potential is then defined as

$$
V_{N F}(y)=\sum_{\beta \in \beta_{0}} c_{\beta} y^{\beta},
$$

so that the scalar normal form Hamiltonian becomes

$$
H_{N F}=-\frac{1}{2} \Delta+V_{N F} .
$$

Taking into account our normalization (3.2), we expect the spectrum of $H_{\mathrm{mol}}(\epsilon)$ to contain elements of the form

$$
\mathcal{E}(\epsilon)=\epsilon^{\sigma} \mathcal{E}_{\sigma}+O\left(\epsilon^{\sigma+\xi}\right),
$$

for small $\epsilon$, where

$$
\mathcal{E}_{\sigma} \in \sigma\left(H_{N F}\right) \quad \text { and } \quad \xi>0 .
$$

The index $\sigma$ refers to the power of $\epsilon$ in the leading term.

Let us briefly consider the existence of a suitable normal form for a given smooth potential of the form (3.4) and, in particular, the definition of the exponent $\sigma>0$. 
Consider the usual situation in which at least one monomial in the Taylor expansion (3.4) whose coefficient is independent of $\epsilon$ appears in the normal form. Such terms must define the exponent $\sigma$, because they contain no extra $\epsilon$ dependence, and after rescaling, their contribution to the normal form cannot diverge. Thus, we introduce the set

$$
\beta_{N}=\left\{\beta \in \mathbb{N}^{n}: v_{\beta}(\epsilon)=c_{\beta} \neq 0\right\} .
$$

The dimensions corresponding to the minimal value $\mu_{n}$ of the exponents $\mu_{j}$ play a special role. Let $0 \leq k \leq n$ be the integer such that $\mu_{n}=\mu_{n-1}=\cdots \mu_{n-k}<$ $\mu_{n-k-1}$. We define

$$
\beta_{\|}=\left\{\beta \in \mathbb{N}^{n}: \beta=\left(0, \cdots, 0, \beta_{n-k}, \beta_{n-k+1}, \cdots, \beta_{n}\right) \equiv\left(0, \cdots, 0, \beta^{\prime \prime}\right)\right\} .
$$

Similarly, we write $x^{\prime \prime}=\left(0, \cdots, 0, x_{n-k}, x_{n-k+1}, \cdots, x_{n}\right)$. For simplicity, we assume that there exists a non-zero term of the form $x^{\prime \beta^{\prime \prime}}$ in (3.4) with an $\epsilon$ independent coefficient. Note, however, that it is possible to prove results similar to the following one in case this hypothesis does not hold.

The next proposition gives conditions on the $\epsilon$-dependence of the coefficients $v_{\beta}(\epsilon)$ (with $\beta \notin \beta_{N}$ ), ensuring that Assumption 3.3 holds, with an exponent $\sigma$ defined by means of the indices of the $\epsilon$-independent non-zero coeffcients of (3.4).

Proposition 3.7. Assume $\beta_{N} \cap \beta_{\|} \neq \emptyset$. Then Assumption 3.3 holds with

$$
\begin{aligned}
\sigma & =\min _{\beta \in \beta_{N}} \frac{\beta \cdot \mu}{2+|\beta|}<\mu_{n}, \\
\beta_{0} & =\left\{\beta \in \mathbb{N}^{n}: \beta \cdot \mu-\sigma(2+|\beta|) \leq 0\right\}, \quad \text { and } \\
\beta_{F} & =\left\{\beta \in \mathbb{N}^{n} \backslash \beta_{0}:|\beta|<F\right\}
\end{aligned}
$$

for some sufficiently large $F>0$, provided

$$
v_{\beta}(\epsilon)\left(\epsilon^{1 / 2}\right)^{\beta \cdot \mu-\sigma(|\beta|+2)}=c_{\beta}, \quad \forall \beta \in \beta_{0} \backslash \beta_{N} .
$$

REMARK 3.8. Actually, $F=\max _{\beta \in \beta_{0}}|\beta|+1$ will do.

Proof. Observe that for $|\beta|$ fixed,

$$
\frac{\beta \cdot \mu}{2+|\beta|} \geq \frac{|\beta| \mu_{n}}{2+|\beta|}
$$

with equality if and only if $\beta \in \beta_{\|}$. Since $x \mapsto \frac{x \mu_{n}}{2+x}$ is strictly increasing, we have

$$
\mu_{j} \geq \mu_{n}>\min _{\beta^{\prime \prime} \in \beta_{N} \cap \beta_{\|}} \frac{\left|\beta^{\prime \prime}\right| \mu_{n}}{2+\left|\beta^{\prime \prime}\right|} \geq \sigma .
$$

Moreover, this shows that the minimum over $\beta_{N}$ is reached on the finite set $\{\beta \in$ $\left.\mathbb{N}^{n}:|\beta| \leq \min _{\beta^{\prime \prime} \in \beta_{\|}}\left|\beta^{\prime \prime}\right|\right\}$. Then, Remark 3.6 implies that $\beta_{0}$ is finite. Moreover, for any $\beta \in \beta_{N}, \quad \beta \cdot \mu-\sigma(2+|\beta|) \geq 0$. Hence, the corresponding terms in the Taylor series either contribute to the normal form, or they are of higher order and can be neglected. The last condition ensures that the $\epsilon$ dependence of the coefficients is strong enough to compensate for the negative exponents $\beta \cdot \mu-\sigma(|\beta|+2)$. 


\section{Mathematical properties of $H_{N F}$}

The normal form Hamiltonian $H_{N F}$ plays the same role that the harmonic oscillator plays in the standard Born-Oppenheimer approximation. However, in contrast with the harmonic oscillator, we do not know the spectral properties of $H_{N F}$ explicitly. In [3] we prove certain properties that we require for our analysis. We now describe them.

We consider Schrödinger operators with potentials that satisfy the following hypothesis.

Assumption 4.1. Let $H=-\frac{1}{2} \Delta+V$ be self-adjoint on a suitable domain in $L^{2}\left(\mathbb{R}^{n}\right)$, where $x \mapsto V(x)$ is a polynomial and $V \geq 0$.

The first concern is the spectrum of the normal form Hamiltonian. We want purely discrete spectrum, which is not obvious with our polynomial potentials $V$ that may or may not be confining. Depending on the parameters, the set $V^{-1}(\{0\})$ may be unbounded. In order to exclude essential spectrum, we could use Persson's Theorem, as we did in [3] for the tri-atomic case. A more general criterion for the absence of essential spectrum for Schrödinger operators with polynomial potentials can be found in $[\mathbf{7}]^{1}$. Within our framework, it states the following:

For a polynomial $V \geq 0$, define

$$
m_{V}^{*}(x)=1+\sum_{\alpha \in \mathbb{N}^{n}}\left|D^{\alpha} V(x)\right|,
$$

where $D^{\alpha}=\partial_{x_{1}}^{\alpha_{1}} \partial_{x_{2}}^{\alpha_{2}} \cdots \partial_{x_{n}}^{\alpha_{n}}$. Note that the sum is finite.

Proposition 4.2 ([7], Thm (1.3)). Let $H$ satisfy Assumption 4.1 and let $m_{V}^{*}$ be defined by (4.1). Then, the resolvent of $H$ is compact if and only if

$$
\lim _{|x| \rightarrow \infty} m_{V}^{*}(x)=\infty
$$

REMARK 4.3. The proof of this result in $[\mathbf{7}]$ finds its roots in earlier work on hypoelliptic operators. See e.g. [5].

The function $m_{N F}^{*}$ corresponding to the normal form Hamiltonian for tri-atomic molecules, i.e., to the potential $E_{\mathrm{NF}}\left(x_{1}, x_{2}\right)$ given by

$$
E_{\mathrm{NF}}\left(x_{1}, x_{2}\right)=a_{1} x_{1}^{2}+\left(a_{2}-a_{3} x_{1}\right) x_{2}^{2}+a_{4} x_{2}^{4},
$$

is easily seen to tend to infinity as $|x|$ tends to infinity if Assumption 2.1 holds Hence, $\sigma\left(H_{N F}\right)$ is discrete, for all allowed values of the parameters $\left\{a_{j}\right\}$.

For the general normal form potential $V_{N F}$ (3.7), we assume that the corresponding function $m_{N F}^{*}$ diverges at infinity.

We also need to know that the eigenfunctions decay exponentially, in a $L^{2}$ sense, together with their derivatives. This is the content of the next result which is proven in [3].

\footnotetext{
${ }^{1}$ We thank B. Helffer for pointing this criterion out to us.
} 
Proposition 4.4. Let $H$ satisfy Assumption 4.1, and assume that the spectrum of $H$ is discrete. Then, any eigenfunction $x \mapsto \varphi(x) \in L^{2}\left(\mathbb{R}^{n}\right)$ is $C^{\infty}$ and, for any $a>0$,

$$
\varphi \in D\left(e^{a\langle x\rangle}\right), \quad \nabla \varphi \in D\left(e^{a\langle x\rangle}\right), \quad \text { and } \quad \Delta \varphi \in D\left(e^{a\langle x\rangle}\right),
$$

where $\langle x\rangle=\left(1+\sum_{j=1}^{n} x_{j}^{2}\right)^{1 / 2}$, and $D\left(e^{a\langle x\rangle}\right)$ denotes the domain of the operator multiplication by $e^{a\langle x\rangle}$.

Proof. The smoothness of the eigenfunctions follows from elliptic regularity arguments. Their exponential decay properties are consequences of classical Combes-Thomas arguments. Since the potential is a polynomial, we immediately deduce the exponential decay of $\Delta \varphi$ by using the eigenvalue equation. That $\nabla \varphi \in D\left(e^{a\langle x\rangle}\right)$ is a consequence of the following lemma, with $p(x)=e^{a\langle x\rangle}$ :

LEMmA 4.5. Let $x \mapsto p(x) \in C^{1}\left(\mathbb{R}^{n}\right)$ be positive, and assume that there exists a constant $C<\infty$, such that $|(\nabla p(x)) / p(x)| \leq 2 C$ for all $x \in \mathbb{R}^{n}$.

Then, for any $f \in\left\{g: \int_{\mathbb{R}^{n}}\left(|g(x)|^{2}+|\bar{\Delta} g(x)|^{2}\right) p(x) d x<\infty\right\}$

$$
\left\|p^{1 / 2} \nabla f\right\| \leq C\left\|p^{1 / 2} f\right\|+\sqrt{\left\|p^{1 / 2} f\right\|\left\|p^{1 / 2} \Delta f\right\|+C^{2}\left\|p^{1 / 2} f\right\|^{2}} .
$$

The rather technical proof of this lemma can be found in $[\mathbf{3}]$.

Remark 4.6. While Proposition 4.4 is sufficient to derive the leading contribution (1.2) of the vibrational spectrum, the derivation of a complete asymptotic expansion in powers of $\epsilon^{1 / 2}$ requires a generalization to arbitrary derivatives of the eigenfunction:

Proposition 4.7. Let $H$ satisfy Assumption 4.1 and have discrete spectrum. Then any eigenfunction $x \mapsto \varphi(x) \in L^{2}\left(\mathbb{R}^{n}\right)$ is $C^{\infty}$ and, for any $a>0$, and any multi-index $\gamma \in \mathbb{N}^{n}, \quad D^{\gamma} \varphi \in D\left(e^{a\langle x\rangle}\right)$.

This result is proved by induction on the order of $\gamma$. The initial step is provided by Proposition 4.4. The induction step involves Paley-Wiener arguments. See [3].

REMARK 4.8. We also note that while the exponential decay properties of eigenfunctions of Schrödinger operators have been the object of many detailed and refined investigations (See e.g., [6] ), we could not find any published results that dealt with the decay properties of their successive derivatives.

\section{Leading order results}

In this last section, we show how leading order results of the type (1.2), or more generally, of the type (3.8) can be obtained by means of rather explicit quasimodes. The following theorem is the main result of this note. It is the generalization of Theorem 3.6 in $[\mathbf{3}]$ to the situation described in Section 3.

THEOREM 5.1. Let $H_{\text {mol }}(\epsilon)$ be defined by (3.1) and satisfy Assumption 3.1. Assume the ground state $V(\epsilon, x)$ satisfies Assumption 3.3, and let $H_{N F}$ be the normal form Schrödinger operator $H_{N F}=-\frac{1}{2} \Delta+V_{N F}$, with $V_{N F}$ defined by (3.7). Further assume that the polynomial $V_{N F}$ is bounded below, and that $H_{N F}$ has compact resolvent.

Let $f \in L^{2}\left(\mathbb{R}^{n}\right)$ be a normalized eigenvector corresponding to an eigenvalue $\mathcal{E}_{\sigma}$ of $H_{N F}$

$$
\left(-\frac{1}{2} \Delta_{y}+V_{N F}(y)\right) f(y)=\mathcal{E}_{\sigma} f(y)
$$


Then, for small $\epsilon$, there exists an element $\mathcal{E}(\epsilon)$ of the spectrum of $H_{m o l}(\epsilon)$ that satisfies

for some $\xi>0$.

$$
\mathcal{E}(\epsilon)=\epsilon^{\sigma} \mathcal{E}_{\sigma}+O\left(\epsilon^{\sigma+\xi}\right) .
$$

REMARK 5.2. The tri-atomic results are direct consequences of this result, up to a rescaling of the energy.

REMARK 5.3. Theorem 5.1 applies in particular to scalar Schrödinger operators with potential $V(\epsilon)$ satisfying Assumption 3.3.

REMARK 5.4. In the case of the tri-atomic molecule, it is possible to go beyond leading order results. Theorem 3.7 in $[\mathbf{3}]$ states the existence a complete asymptotic expansion in powers of $\epsilon^{1 / 2}$ for $\mathcal{E}(\epsilon)$.

Proof. We construct a quasimode based on the elements above. This quasimode is supported close to the origin of the coordinates, and we introduce a cutoff function $\mathcal{F}$ on $\mathbb{R}^{n}$, defined as follows:

$$
\mathcal{F}\left(z_{1}, \cdots, z_{n}\right)=\mathcal{F}\left(z_{1}\right) \mathcal{F}\left(z_{2}\right) \cdots \mathcal{F}\left(z_{n}\right)
$$

where $\mathcal{F}(\cdot) \in C^{\infty}(\mathbb{R},[0,1])$ is even, supported on $[-2,2]$, and equal to 1 on $[-1,1]$. We introduce a set of exponents $\left(\delta_{1}, \cdots, \delta_{n}\right)$ with

$$
0<\delta_{j}<\alpha_{j}, \quad \forall j=1,2, \cdots, n,
$$

whose values will be determined in the course of the proof. We also use the shorthand notation $x / \epsilon^{\delta}=\left(x_{1} / \epsilon^{\delta_{1}}, \cdots, x_{n} / \epsilon^{\delta_{n}}\right)$.

Our quasimode is then defined as

$$
\Psi_{Q}(\epsilon, x)=\Phi(x) f\left(x / \epsilon^{\alpha}\right) \mathcal{F}\left(x / \epsilon^{\delta}\right),
$$

where $\Phi(x) \in \mathcal{H}_{e l}$ is the normalized electronic eigenvector corresponding to $V(\epsilon, x)$. Consider its norm,

$$
\left\|\Psi_{Q}(\epsilon)\right\|^{2}=\int_{\mathbb{R}^{n}}\left|f\left(x / \epsilon^{\alpha}\right)\right|^{2} d x-\int_{\mathbb{R}^{n}}\left(1-\mathcal{F}^{2}\left(x / \epsilon^{\delta}\right)\right)\left|f\left(x / \epsilon^{\alpha}\right)\right|^{2} d x .
$$

The first term is equal to $\epsilon^{|\alpha|}$ by scaling. Using the exponential decay of $f$, Proposition 4.4, and the constraint (5.3) on the exponents, we see that the negative of the second term is bounded above by

$$
\begin{aligned}
\int_{\left|x_{j}\right| \geq \epsilon^{\delta_{j}}}\left|f\left(x / \epsilon^{\alpha}\right)\right|^{2} d x & \leq \epsilon^{|\alpha|} \int_{\left|y_{j}\right| \geq \epsilon^{\delta_{j}-\alpha_{j}}} e^{-2 a \sum_{j=1}^{n}\left|y_{j}\right|} e^{2 a \sum_{j=1}^{n}\left|y_{j}\right|}|f(y)|^{2} d y \\
& \leq \epsilon^{|\alpha|} e^{-2 a \sum_{j=1}^{n} 1 / \epsilon^{\alpha_{j}-\delta_{j}}}\left\|e^{a \sum_{j=1}^{n}\left|y_{j}\right|} f\right\|^{2} \\
& =O\left(\epsilon^{\infty}\right) .
\end{aligned}
$$

Altogether, this shows that $\left\|\Psi_{Q}(\epsilon, x)\right\|=\epsilon^{|\alpha| / 2}\left(1+O\left(\epsilon^{\infty}\right)\right)$. Thus, to prove the result, we need only show that

$$
\left\|H_{\mathrm{mol}}(\epsilon) \Psi_{Q}(\epsilon)-\epsilon^{\sigma} \mathcal{E}_{\sigma} \Psi_{Q}(\epsilon)\right\|=O\left(\epsilon^{|\alpha| / 2+\sigma+\xi}\right),
$$

for some $\xi>0$. Using (3.1) and the definitions (3.7), (5.1), we see that

$$
\begin{aligned}
H_{\mathrm{mol}}(\epsilon) \Psi_{Q}(\epsilon)-\epsilon^{\sigma} \mathcal{E}_{\sigma} \Psi_{Q}(\epsilon)= & \left(V(\epsilon, x)-\epsilon^{\sigma} V_{N F}\left(x / \epsilon^{\alpha}\right)\right) \Psi_{Q}(\epsilon, x) \\
& -\mathcal{R}(\epsilon, x),
\end{aligned}
$$


where

$$
\begin{aligned}
\mathcal{R}(\epsilon, x) & =\sum_{j=1}^{n} \epsilon^{\mu_{j}} \Phi(x) f\left(x / \epsilon^{\alpha}\right) \partial_{x_{j}}^{2} \mathcal{F}\left(x / \epsilon^{\delta}\right) / 2 \\
& +\sum_{j=1}^{n} \epsilon^{\mu_{j}} \partial_{x_{j}}\left(\Phi(x) f\left(x / \epsilon^{\alpha}\right)\right) \partial_{x_{j}} \mathcal{F}\left(x / \epsilon^{\delta}\right) \\
& +\sum_{j=1}^{n} \epsilon^{\mu_{j}}\left(f\left(x / \epsilon^{\alpha}\right) \partial_{x_{j}}^{2} \Phi(x) / 2+\partial_{x_{j}} f\left(x / \epsilon^{\alpha}\right) \partial_{x_{j}} \Phi(x)\right) \mathcal{F}\left(x / \epsilon^{\delta}\right) .
\end{aligned}
$$

We consider the first two terms in $\mathcal{R}(\epsilon)$, where $\Phi$ and its derivatives are uniformly bounded in a neigborhood of the origin. Because of the derivatives of the cutoff function, there is always one direction in which the integration range is away from the origin. Using this together with the fact that $f$ and $\partial_{y_{j}} f$ are exponentially decaying in any direction, an argument similar to that used in (5.6) shows that these contributions are $O\left(\epsilon^{\infty}\right)$. The last contribution to $\mathcal{R}(\epsilon)$ is simply estimated by $O\left(\sum_{j=1}^{n} \epsilon^{|\alpha| / 2} \epsilon^{\mu_{j}-\alpha_{j}}\right)$, where $\epsilon^{|\alpha| / 2}$ comes from scaling. By $(3.3), \mu_{j}-\alpha_{j}=$ $\sigma+\alpha_{j}>\sigma$, so that $\mathcal{R}(\epsilon)$ satisfies the bound (5.7). To bound the norm of the first term in (5.8), we use (3.5) and (3.7) to see that

$$
\begin{aligned}
\left\|\left(V(\epsilon)-\epsilon^{\sigma} V_{N F}\left(\cdot / \epsilon^{\alpha}\right)\right) \Psi_{Q}(\epsilon)\right\| & \leq c \sum_{\beta \in \beta_{F}}\left(\int_{\left|x_{j}\right| \leq 2 \epsilon^{\delta_{j}}}\left|x^{\beta}\right|^{2}\left|f\left(x / \epsilon^{\alpha}\right)\right|^{2} d x\right)^{1 / 2} \\
& \leq \tilde{c} \epsilon^{|\alpha| / 2} \epsilon^{\beta \cdot \delta},
\end{aligned}
$$

for some constant $\tilde{c}$. By assumption, for all $\beta \in \beta_{F}$, we have

$\beta \cdot \alpha-\sigma=\frac{1}{2}(\beta \cdot \mu-\sigma(|\beta|+2))>0$. Hence, by continuity, choosing $\delta_{j}<\alpha_{j}$ close to $\alpha_{j}$, we get

$$
\epsilon^{|\alpha| / 2} \epsilon^{\beta \cdot \delta}=\epsilon^{|\alpha| / 2+\sigma} \epsilon^{\beta \cdot \delta-\sigma} \equiv \epsilon^{|\alpha| / 2+\sigma} \epsilon^{\xi}, \quad \text { with } \quad \xi>0
$$

which ends the proof.

REMARK 5.5. From the proof, we get that the quasimode for the tri-atomic molecule is of the form

$$
\Psi_{Q}\left(\epsilon, x_{1}, x_{2}\right)=\Phi\left(x_{1}, x_{2}\right) f\left(x_{1} / \epsilon, x_{2} / \epsilon^{1 / 2}\right) \mathcal{F}\left(x_{1} / \epsilon^{\delta_{1}}, x_{2} / \epsilon^{\delta_{2}}\right),
$$

for suitable values of $\delta_{1}$ and $\delta_{2}$, and where $f$ is an eigenfunction of the normal form (2.3). Provided we have further arguments supporting that fact that quasimodes are close to true eigenvectors, we observe the following. The amplitude of the distance between the two heavy nuclei is of order $\epsilon$, whereas the hydrogen atom can move a larger distance away form its equilibrium position, i.e., of order $\sqrt{\epsilon}$.

\section{References}

[1] M. Born and J. R. Oppenheimer, Zur Quantentheorie der Molekeln. Ann. Phys. (Leipzig) 84 (1927), 457-484.

[2] N. Elghobashi, Theory of using few cycles IR and UV laser pulses to control the orientation and selective dissociation of Hydrogen-bonded anions, Ph D dissertation, Freie Universität Berlin, (2005).

[3] G.A. Hagedorn, A. Joye, A Mathematical Theory for Vibrational Levels Associated with Hydrogen Bonds I: The Symmetric Case, Commun. Math. Phys. (to appear). 
[4] G.A. Hagedorn, A. Joye, Mathematical Analysis of Born-Oppenheimer Approximations, in Spectral Theory and Mathematical Physics: A Festschrift in Honor of Barry Simon's 60th Birthday. Part I: Quantum Field Theory, Statistical Mechanics, and Nonrelativistic Quantum Systems, F. Gesztesy, P. Deift, C. Galvez, P. Perry, W. Schlag, Edts, AMS Proc. of Symposia in Pure Math. 76 (2007), 203-226.

[5] B. Helffer, J. Nourrigat, Hypoellipticité maximale pour des opérateurs polynômes de champs de vecteurs, Birkhäser, Boston, 1985.

[6] P. Hislop, Exponential decay of two-body eigenfunctions: A review in "Mathematical Physics and Quantum Field Theory", Henry Warchall (Editor), Electronic J. Diff. Eqns., Conf. 04 (2000), 265-288.

[7] A. Mohamed, J. Nourrigat, Encadrement du $N(\lambda)$ pour un opérateur de Schrödinger avec un champ magnétique et un potentiel électrique, J. Math. Pures Appl. 70 (1990), 87-99.

Department of Mathematics and Center for Statistical Mechanics, Mathematical Physics, and Theoretical Chemistry, Virginia Polytechnic Institute and State UniverSity, Blacksburg, Virginia 24061-0123, U.S.A.

E-mail address: hagedorn@calvin.math.vt.edu

Institut Fourier, Université de Grenoble I, BP 74, F-38402 Saint Martin D'Hères Cedex, France, and, Laboratoire de Physique et Modélisation des Milieux Condensés, Université de Grenoble I, BP 166, F-38042 Grenoble, France

E-mail address: alain.joye@ujf-grenoble.fr 